\title{
Ibrutinib: an evidence-based review of its potential in the treatment of advanced chronic lymphocytic leukemia
}

This article was published in the following Dove Press journal:

Core Evidence

15 May 2013

Number of times this article has been viewed

\author{
Julio C Chavez \\ Eva Sahakian \\ Javier Pinilla-lbarz \\ H Lee Moffitt Cancer and Research \\ Institute, Division of Malignant \\ Hematology, and University of South \\ Florida, Tampa, FL, USA
}

\begin{abstract}
Chronic lymphocytic leukemia (CLL) is a heterogeneous disease with a variable course, and remains an incurable disease. Frequent relapses and eventual resistance to fludarabine characterize symptomatic CLL and portends a dismal prognosis for patients. Growing evidence has shown that signaling pathways such as the B cell receptor and NFkB are implicated in the survival and proliferation of the CLL cells which are ultimately associated with persistence of the disease. The Bruton's tyrosine kinase pathway regulates downstream activation of the $\mathrm{B}$ cell receptor and has emerged as an attractive target. Ibrutinib inhibits the Bruton's tyrosine kinase pathway, and consequently induces apoptosis of B cells. Phase I and II studies have shown impressive response rates with an excellent safety profile in patients with refractory/relapsed CLL and elderly treatment-naïve CLL patients. This paper reviews the preclinical and clinical data for ibrutinib when used in the treatment of CLL. Recent studies showing the benefit of combination therapy using ibrutinib, monoclonal antibodies, and chemoimmunotherapy are also discussed.
\end{abstract}

Keywords: ibrutinib, B-cell receptor, chronic lymphocytic leukemia, Bruton's tyrosine kinase

\begin{tabular}{|c|c|c|}
\hline $\begin{array}{l}\text { Outcome } \\
\text { measure }\end{array}$ & Evidence & Implications \\
\hline $\begin{array}{l}\text { Disease-oriented } \\
\text { evidence }\end{array}$ & $\begin{array}{l}\text { Preclinical studies } \\
\text { BTK inhibition } \\
\text { Inhibition of CXCLI } 2 \text { and CXCLI3 } \\
\text { Inhibition of BAFF, CD } 40, \text { TLR }\end{array}$ & $\begin{array}{l}\text { Induction of B cell apoptosis } \\
\text { Targeting of CLL } \\
\text { microenvironment } \\
\text { Inhibition of CLL homing and } \\
\text { migration }\end{array}$ \\
\hline \multirow[t]{4}{*}{$\begin{array}{l}\text { Patient-oriented } \\
\text { evidence }\end{array}$} & $\begin{array}{l}\text { Phase I study determined the MTD } \\
\text { with minimal side effects. Almost } \\
\text { 100\% BTK inhibition at } 4 \text { hours }\end{array}$ & $\begin{array}{l}\text { Rapid mechanism of action with } \\
\text { acceptable toxicity profile }\end{array}$ \\
\hline & $\begin{array}{l}\text { Phase II: R/R CLL }(n=75) \text {. ORR } 67 \% \text {, } \\
\text { CR } 3 \%\end{array}$ & $\begin{array}{l}\text { Objective response in R/R CLL } \\
\text { including high-risk features }\end{array}$ \\
\hline & $\begin{array}{l}\text { Phase II: elderly treatment-naïve } \\
\text { CLL }(\mathrm{n}=31) . \text { ORR } 73 \% \text {, CR } 8 \%\end{array}$ & $\begin{array}{l}\text { Potential alternative for } \\
\text { elderly CLL patients unfit for } \\
\text { chemotherapy }\end{array}$ \\
\hline & $\begin{array}{l}\text { Phase II: R/R CLL. With rituximab } \\
(n=40) . \text { ORR } 85 \% \\
\text { Phase II: R/R CLL. With ofatumumab } \\
(n=27) . \text { ORR I00\% } \\
\text { Phase II: CLL fludarabine-naïve. With } \\
\text { BR. ORR } 93 \%(C R \text { I3\%) }\end{array}$ & $\begin{array}{l}\text { Impressive response in combination } \\
\text { with monoclonal antibodies } \\
\text { Impressive response in combination } \\
\text { with chemoimmunotherapy with } \\
\text { acceptable toxicity profile }\end{array}$ \\
\hline
\end{tabular}

Correspondence: Javier Pinilla-lbarz I 2902 Magnolia Drive, Tampa,

FL 336 I2, USA

Tel +I 8I3745 2069

Fax +18137454064

Email javier.pinilla@moffitt.org 


\begin{tabular}{|ll|}
\hline $\begin{array}{l}\text { (Continued) } \\
\text { Outcome } \\
\text { measure }\end{array}$ & Evidence \\
\hline $\begin{array}{l}\text { Economic } \\
\text { evidence }\end{array}$ & Unknown as ibrutinib has not been \\
\hline $\begin{array}{l}\text { Abbreviations: BTK, Bruton's tyrosine kinase; CLL, chronic lymphocytic leukemia; R/R, relapsed/refractory; } \\
\text { ORR, overall response rate; CR, complete remission; BR, bendamustine+ rituximab; MTD, maximum tolerated } \\
\text { dose; TLR, Toll-like receptor. }\end{array}$ \\
\hline
\end{tabular}

\section{Introduction}

Chronic lymphocytic leukemia (CLL) is the most common chronic leukemia in Western countries and is most prevalent in the elderly, with a median age of 72 years at diagnosis. ${ }^{1}$ CLL is a malignancy of mature B cells which is most often diagnosed incidentally as a peripheral lymphocytosis. It follows a heterogeneous and variable course, ranging from an indolent disease to a most aggressive clinical pattern with intrinsic resistance to chemotherapy, eventually leading to death. So far, there have been significant advances in the treatment of CLL with the discovery of new prognostic factors and development of highly active chemoimmunotherapy regimens, with good results in terms of progression-free and overall survival. However, with standard therapy, CLL remains an incurable disease, where patients eventually progress to advanced disease followed by short overall survival after several regimens or as a result of refractoriness to chemotherapy. ${ }^{2-5}$ Therefore, there is an immediate need for more specific therapies targeting pathways involved in development, proliferation, and survival of B cells, that are imperative in the pathogenesis of CLL.

\section{Biology of CLL}

CLL represents a monoclonal expansion of mature CD5+, CD23+ B lymphocytes in the blood, lymph nodes, and bone marrow. The disease is selected through multiple expositions to (auto) antigens that differ in degree of gene mutation in the immunoglobulin heavy chain variable region. ${ }^{6}$ Nonmutated heavy chain variable region, pregerminal center, and germinal center-independent B cells usually follow a more aggressive course than their germinal center, post-germinal center, or mutated heavy chain variable region-mutated cell counterparts. In the last few years, major progress in the field has expanded our knowledge and given us a better understanding of the biology, molecular pathogenesis, and mechanism of resistance to treatment in CLL. Various factors are associated with the development, proliferation, and survival of malignant B cells in CLL, such as chronic $B$ cell receptor (BCR) activation, interaction with the microenvironment, chronic (auto) antigen stimulation, and acquisition of genetic lesions. ${ }^{7,8}$ Immune tolerance has also been postulated as key to the survival of B cells in CLL, by maintaining self-reactive BCR and thus continuous activation. ${ }^{9}$ It is known that CLL cells not only depend on intrinsic pathways but also that they are supported by extrinsic signals provided by the tissue microenvironment. These signals are generally comprised of T cells, stromal cells, and many tissue factors, including cytokines, chemokines, CD40, integrins, Toll-like receptors, and other components of the stroma matrix. ${ }^{10}$

\section{$B-c e l l$ receptor and signaling}

The BCR is a key component of normal B cell development and is also implicated in the development of the most common B cell malignancies, including diffuse large B cell lymphoma, follicular lymphoma, mantle cell lymphoma, and CLL. BCR regulates multiple cellular processes, including proliferation, differentiation, apoptosis, and cell migration, which are essential for the functioning and survival of both normal and malignant B cells. ${ }^{11,12}$

The BCR consists of a transmembrane immunoglobulin (Ig) receptor associated with Ig-alpha/Ig-beta heterodimers (CD79a/CD79b) (Figure 1). Antigen binding induces phosphorylation of immunoreceptor tyrosine-based activation motifs located in the cytoplasmic portion of the IgM by the Src family kinases, ie, LYN and the spleen tyrosine kinase (SYK), initiating a cascade of events downstream that involve activation of other kinases and second messengers. ${ }^{13-15}$ Among those signaling molecules is Bruton's tyrosine kinase (BTK), which is essential in the BCR signaling pathway, along with molecules such as mammalian target of rapamycin (mTOR), phosphoinositide 3'-kinase (PI3K), SYK, LYN, the adaptor protein $\mathrm{GrB} 2$, and the B cell linker. ${ }^{14,16}$ Essentially, activated BTK and PI3K induce calcium mobilization and activation of $\mathrm{mTOR}$, protein kinase $\mathrm{C}$-beta, AKT kinase, and mitogen-activated protein kinase ERK. These events result in increased proliferation and survival of B cells, mediated by upregulation of transcription factors, mainly nuclear factor-kB (NFkB). ${ }^{11,13}$

As mentioned above, the microenvironment of the lymph nodes and bone marrow plays an important role in the pathogenesis of CLL by promoting further proliferation 


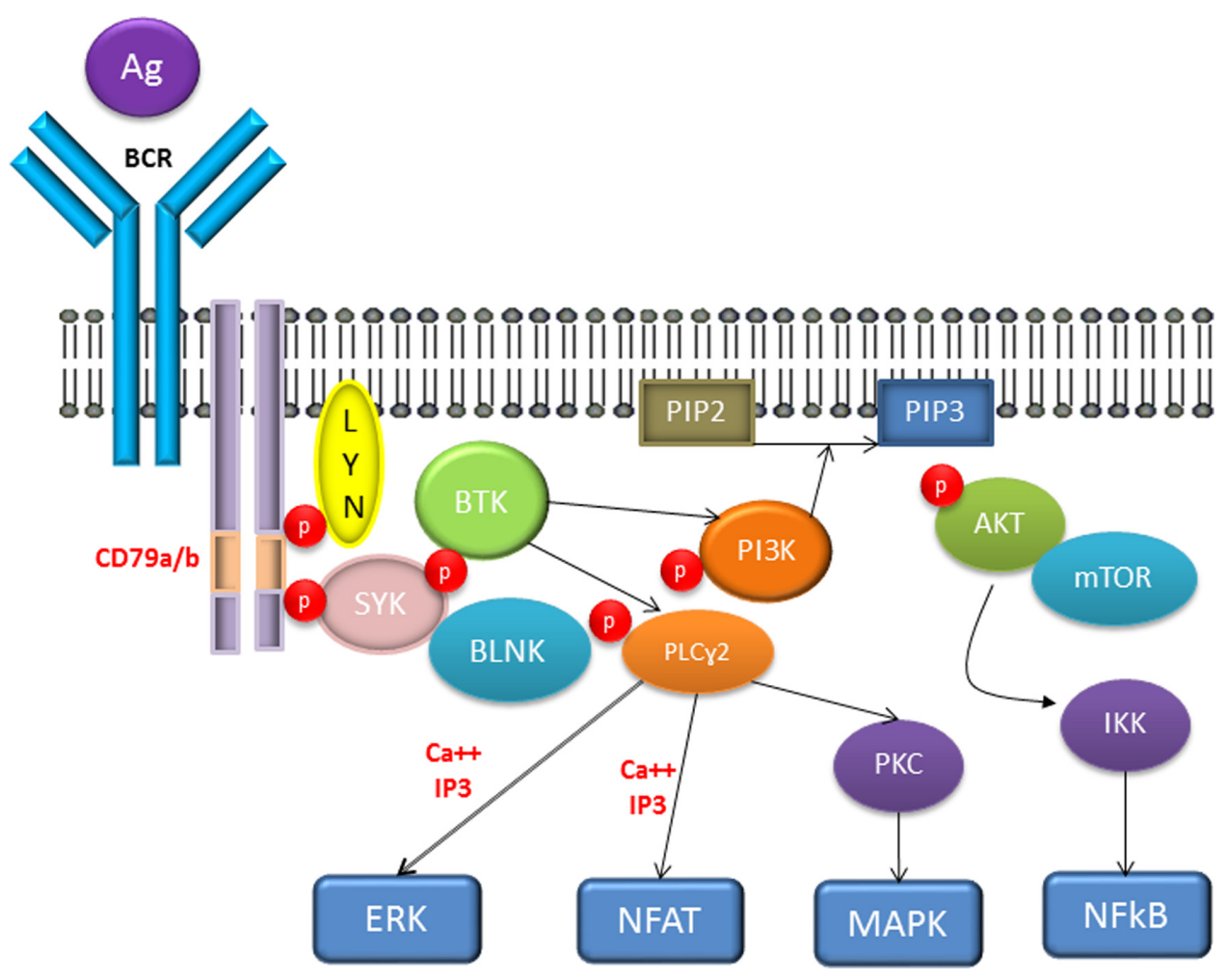

Figure I The $B C R$ pathway.

Abbreviations: AKT, protein kinase B; BCR, B-cell receptor; BLNK, B cell linker protein; BTK, Bruton's tyrosyne kinase; ERK, extracellular regulated kinase; IKK, inhibitor of kB kinase; LYN, Src family tyrosine kinase; MAPK, mitogen activated protein kinase; mTOR, Mammalian target of rapamycin; NFAT, nuclear factor of activated t cells; NFkB, nuclear facto kB; PI3K, phosphatidyl inositol-3 kinase; PLCy2, phospholipase cy2; PKC, protein kinase C; SYK, spleen tyrosine kinase.

and survival. This is achieved by complex mechanisms in which enhanced BCR activation is tightly implicated. On activation of BCR signaling, subsequent BTK and PI3K activations occur which consequently manifest in upregulation of CLL-specific chemokines, such as CCL3 and CCL4, which activate $T$ cells, generating survival signals via CD40/CD40L interactions. ${ }^{17}$ Nurse-like cells are stromal cells that promote survival through a series of factors, including APRIL, BAFF, and CD31 (the latter interacts with CD38 and ZAP-70 to enhance proliferative effects). Lastly, bone marrow stromal cells enhance CLL survival by direct interaction with cells (via CD49d) and by producing mediators such as CXCL12 and CXCL13, which recruit CLL cells, ultimately promoting chemotaxis and homing into the microenvironment. ${ }^{14,18}$ In addition to antigen-driven BCR activation, there is ligandindependent (tonic) signaling activation which is postulated to contribute to the pathogenesis of CLL and is mediated by PI3K-alpha and PI3K-delta. ${ }^{19,20}$

With recent studies demonstrating impressive efficacy in targeting the different mediators of BCR signaling, the importance of this signaling cascade has become the rationale behind the increased interest in targeting key players in this pathway. Among these, an SYK inhibitor (fostamatinib) and a selective PI3K-delta inhibitor (GS-1101, idelalisib) have been studied, with promising data emerging in recent clinical trials. ${ }^{21-25}$

\section{Biology of BTK pathway}

BTK is a cytoplasmic protein and a member of the Tec family of kinases, that is predominantly expressed on B lymphocytes, lymphocyte precursors, and developing myeloid cells, but is absent in plasma cells and T cells. ${ }^{26}$ The role of BTK in BCR activation was first discovered when BTK mutations were found in patients with X-linked Bruton's agammaglobulinemia leading to marked $\mathrm{B}$ cell dysfunction and recurrent severe bacterial infections. ${ }^{27}$ Loss of the Btk gene causes inhibition of B cell lymphocyte function which leads to impaired production of all classes of immunoglobulins, in addition to a complete absence of B cells. ${ }^{28}$

Upon activation of BCR, PI3K is activated, which in turn stimulates production of phosphatidylinositol-3,4,5 (PIP3). Once a sufficient amount of PIP3 is produced, BTK is recruited to the plasma membrane. BTK then undergoes phosphorylation at the Y551 site by Src family kinases, especially LYN and FYN. ${ }^{29,30}$ Phosphorylated BTK activates phospholipase Cy2, leading to downstream activation of protein kinases (such as protein kinase C-beta) and, finally, activation of transcription factor NFkB. 
Stimulation of the NFkB pathway leads to inhibition of the apoptosis machinery, and further facilitates stimulation of the Btk gene. ${ }^{31}$ This series of events has been linked to proliferation and increased survival of lymphoid malignancies, especially CLL. ${ }^{30,32}$

\section{Preclinical studies}

Ibrutinib (formerly PCI-32765) is an orally administered, specific, irreversible, and highly potent BTK inhibitor. It binds covalently to a cysteine-481 residue at the active site of BTK, resulting in potent inhibition of kinase activity, with an $\mathrm{IC}_{50}$ of $0.5 \mathrm{nM}$ for more than 24 hours. ${ }^{33}$

Initial in vitro studies by Honiberg et al using mouse models of rheumatoid arthritis and lupus demonstrated that selective inhibition of BTK leads to blockade of B cell signaling. Ibrutinib inhibited autophosphorylation of BTK $\left(\mathrm{IC}_{50}, 11 \mathrm{nM}\right)$, phosphorylation of PLCy (a physiological substrate of BTK), and phosphorylation of ERK (a downstream kinase). Mouse models of arthritis were treated with varying doses of ibrutinib, and an objective response in clinical arthritis scores was noted, along with a significant reduction in anticollagen autoantibodies. In mouse models of lupus with associated glomerulonephritis, ibrutinib produced a significant reduction in proteinuria, blood urea nitrogen, and anti-double-stranded DNA levels. This study also investigated the efficacy of ibrutinib in a canine model of $\mathrm{B}$ cell lymphoma and showed a striking $70 \%$ reduction in tumor burden. BTK occupancy was tested by monitoring labeled BTK and PCI-33380, and it was determined that a single dose of $2.5-20 \mathrm{mg} / \mathrm{kg}$ was sufficient to occupy BTK fully. ${ }^{33}$

Davis et al studied the effects of ibrutinib in different types of lymphoma cell lines in order to evaluate the implications of blocking BCR signaling in diffuse large B cell lymphoma and the relationship with the signaling adaptor CARD11. CARD11 is required during constitutive activation of the NFkB pathway, which is key to survival of activated B-cell-like diffuse large B cell lymphoma. This study demonstrated that ibrutinib was active in this type of lymphoma with wild-type CARD11, but not in mutant CARD11 cell lines. The germinal center B cell does not rely on the NFkB pathway, so no activity was demonstrated with the BTK inhibitor. ${ }^{33,34}$

Given the results described above, ibrutinib was tested in CLL cell lines. In an early study, Herman et al demonstrated dose-dependent and time-dependent cytotoxic effects of ibrutinib in CLL. Apoptosis mediated by the caspase pathway was seen as early as 12 hours after exposure to the drug. Although cell death induced by ibrutinib was selective for
Table I Clinical trial of ibrutinib in chronic lymphocytic leukemia

\begin{tabular}{|c|c|c|c|c|c|}
\hline Study & $\mathbf{n}$ & $\begin{array}{l}\text { Median follow-up } \\
\text { (months) }\end{array}$ & ORR & CR & $\mathbf{P R}+\mathbf{L}$ \\
\hline \multicolumn{6}{|l|}{ Single agent } \\
\hline $\mathrm{R} / \mathrm{R} \mathrm{CLL}$ & 61 & 17.3 & $67 \%$ & $3 \%$ & $20 \%$ \\
\hline TN elderly & 31 & 16.6 & $71 \%$ & $10 \%$ & $10 \%$ \\
\hline High-risk & 24 & 10.3 & $50 \%$ & 0 & $29 \%$ \\
\hline \multicolumn{6}{|l|}{ Combination } \\
\hline Plus BR & 30 & 8.1 & $93 \%$ & $13 \%$ & $3 \%$ \\
\hline Plus rituximab & 40 & 4.1 & $83 \%$ & $3 \%$ & $8 \%$ \\
\hline Plus ofatumumab & 27 & 6.5 & $100 \%$ & $4 \%$ & NR \\
\hline
\end{tabular}

Abbreviations: CLL, chronic lymphocytic leukemia; R/R, relapsed/refractory; $\mathrm{TN}$, treatment-naïe; $\mathrm{ORR}$, overall response rate; $\mathrm{CR}$, complete remission; $P R+L$, partial remission plus lymphocytosis; BR, bendamustine + rituximab; $\mathrm{NR}$, not reported.

$\mathrm{B}$ cells, it also altered the immunogenicity of $\mathrm{T}$ cells by inhibiting production of interleukins 6 and 10 and tumor necrosis factor-alpha by $\mathrm{T}$ cells without affecting survival. This study also demonstrated reduced signaling in other BCR-dependent pathways, such as mitogen-activated protein kinase, PI3K, and NFkB, by testing phosphorylation status of intermediate molecules such as AKT and ERK 1/2. Ibrutinib also affected the CLL microenvironment by inhibiting CD40, BAFF, Toll-like receptor, and cytokine signaling, thereby disrupting the protective effects of stromal cells. ${ }^{35}$

Another study by Ponader et al focused on the CLL microenvironment and demonstrated decreased viability of CLL cell lines, cocultures with nurse-like cells, in response to inhibition of BCR signaling by ibrutinib. ${ }^{36}$ This study also showed disruption of homing and adhesion of CLL tissue to the stroma via reduced secretion of chemokines CCL3 and CCL4, which are secreted in response to activation of BCR. It has been suggested that CLL3 and CLL4 levels could be used as biomarkers of drug activity. Migration and chemotaxis were further disrupted by inhibition of the interaction with CXCL12 and CXCL13. ${ }^{36,37}$

Given the findings discussed thus far, it is very likely that the action of ibrutinib is not only mediated by direct BTK signaling inhibition downstream BCR, but also by affecting the CLL microenvironment and the interaction between stromal cells, cellular adhesion, migration, and homing.

\section{Clinical studies}

Clinical studies of ibrutinib in CLL started in May 2010. The published reports include its use as a single agent in the refractory setting and as upfront therapy in elderly patients. There are also ongoing Phase II trials using ibrutinib in combination with monoclonal antibodies and 
chemoimmunotherapy. These studies are summarized in Table 1.

\section{Ibrutinib as monotherapy}

A Phase I study was started and included 56 patients with relapsed/refractory B cell malignancies, including CLL, non-Hodgkin's lymphomas, and Waldenstrom's macroglobulinemia. The patients received escalating weight-based doses of ibrutinib, with six dose levels, starting at $1.25 \mathrm{mg} / \mathrm{kg}$. The medication was administered on a 35-day cycle schedule (28 days on, seven days off). Pharmacokinetic studies showed that ibrutinib was rapidly absorbed and eliminated, with mean peak plasma concentrations observed 1-2 hours after drug administration. The terminal mean half-life was 4-8 hours, with no evidence of drug accumulation after repeated dosing. No dose-limiting toxicities were observed, and the drug was well tolerated. BTK occupancy was assessed using an assay probe which bound the cysteine residue target in the absence of the drug. More than 90\% BTK occupancy was observed at ibrutinib doses $>2.5 \mathrm{mg} / \mathrm{kg}$, and a $420 \mathrm{mg}$ dose was chosen for future studies. The reported overall response rate was $60 \%$. In 14 patients with CLL, the overall response rate was 70\%, with two complete responses. ${ }^{38}$ Most of the adverse events were grade 1 or 2, with very few being grade 3 or 4 (which included neutropenia and thrombocytopenia), and all being dose-independent. ${ }^{38}$

Encouraging results from the Phase I study in CLL prompted a Phase Ib/II study evaluating the efficacy of ibrutinib in CLL patients, and the findings were presented at successive national meetings as abstracts..$^{39-42}$ The most recent data are for 116 patients who were divided into five cohorts comprising: treatment-naïve CLL patients older than 65 years; relapsed/ refractory CLL patients receiving ibrutinib $420 \mathrm{mg}$ and $840 \mathrm{mg}$; and a cohort of high-risk CLL patients (defined as relapse within two years following chemoimmunotherapy and/or presence of $17 \mathrm{p}$ deletion) who received ibrutinib $420 \mathrm{mg}$ daily. The drug was administered on a 28-day cycle until disease progression or severe toxicity was observed. Treatment was discontinued in seven of the 116 patients across all cohorts.

The relapsed/refractory CLL cohort included 27 patients on $420 \mathrm{mg}$ and 34 patients on $840 \mathrm{mg}$. Their median age was 64 years and the median number of treatments was four for the whole cohort. Bulky lymphadenopathy was present in $54 \%$, and $46 \%$ were refractory to fludarabine. Nonmutated IgVH, 17p deletion, and 11q deletion was documented in $86 \%, 37 \%$, and $40 \%$ of cases, respectively. With a median follow-up of 17.5 months for the $420 \mathrm{mg}$ cohort and
10.3 months for the $840 \mathrm{mg}$ cohort, the overall response rate by International Workshop on CLL criteria was $67 \% .{ }^{43}$ Rates of complete response and partial remission were $3 \%$ and $64 \%$, respectively. More than $50 \%$ of patients showed at least a $50 \%$ reduction in lymphadenopathy (partial remission) with residual lymphocytosis. ${ }^{42}$ Interestingly, as soon as the patients showed a nodal response, they developed a significant increase in their absolute lymphocyte counts, a phenomenon known as redistribution lymphocytosis. Because of the otherwise clinical improvement in lymph node status, it was not considered as clinical progression but rather as a form of response described as "partial remission with lymphocytosis" (more than 50\% reduction in lymphadenopathy with residual lymphocytosis). This response was noted in $20 \%$ of the relapsed/refractory CLL patients. Over time, many of these patients have a more than $50 \%$ decrease in absolute lymphocyte count compared with baseline, so they meet the criteria for partial remission. The current International Working Group CLL response guidelines might need to be modified or adapted in the near future in order to include these new response criteria indicating a beneficial clinical effect of ibrutinib, despite this transient lymphocytosis. ${ }^{44}$

The same phenomenon has been also described in CLL patients treated with SYK and PI3K inhibitors. ${ }^{45,46}$ The etiology of this lymphocytosis is believed to be disruption of CLL cell trafficking and homing, secondary to BCR inhibition, which leads to dislodgement of CLL cells from the tumor microenvironment. As mentioned above, activated BCR signaling promotes integrin-mediated CLL adhesion in the stromal tissue and trafficking through the CLL microenvironment, promoting CLL proliferation and survival.

Thirty-one of these cases were treatment-naïve CLL patients older than 65 years, 26 were on ibrutinib $420 \mathrm{mg}$, and five were on ibrutinib $840 \mathrm{mg}$ daily. Their median age was 71 years and the median follow-up duration for the whole group was 16.6 months. Nonmutated IgVH, 11q deletion, and $17 \mathrm{p}$ deletion was found in $55 \%, 3 \%$, and $7 \%$ of patients in this cohort. The overall response rate by International Workshop on CLL criteria was $71 \%$ (10\% with a complete response and $61 \%$ with partial remission) with a partial remission and lymphocytosis rate of $10 \%$ (less than in the relapsed/ refractory CLL cohort).

The high-risk CLL cohort comprised 24 patients who received ibrutinib $420 \mathrm{mg}$ daily. These patients had a median age of 68 years and a median of four prior treatments. A $17 \mathrm{p}$ deletion and nonmutated $\mathrm{IgVH}$ was found in $30 \%$ and $83 \%$ of these patients, respectively. With a median follow-up 
of 10.3 months, the overall response rate by International Workshop on CLL criteria was 50\% (all partial remissions), with $29 \%$ achieving partial remission with lymphocytosis. Four percent of patients progressed while on treatment.

In terms of survival, the estimated 22-month progressionfree survival for the relapsed/refractory and high-risk CLL group was $76 \%$ and for the treatment-naïve group was $96 \%$. Estimated 22-month overall survival was $85 \%$ and $96 \%$ for the relapsed/refractory and treatment-naïve patients, respectively. Interestingly, median progressionfree survival and overall survival have not been reached in all cohorts, including high-risk CLL patients with the $17 \mathrm{p}$ deletion $(57 \%)$.

Of note, the side effects reported were mostly grade 1 to 2 , and consisted of diarrhea (54\%), fatigue (29\%), upper respiratory infection $(29 \%)$, rash $(28 \%)$, nausea $(26 \%)$, and joint pain $(25 \%)$. Hematologic toxicity higher than grade 2 was relatively rare and consisted mostly of neutropenia and thrombocytopenia. Seven of 116 patients discontinued treatment because of adverse events. No fatal events were reported.

\section{Ibrutinib as combination therapy}

The promising results of ibrutinib as monotherapy in refractory and treatment-naïve CLL patients led investigators to explore its efficacy when used in combination with drugs or regimens known to be effective in CLL, with the prospect of enhanced and durable responses.

\section{Ibrutinib and monoclonal antibodies}

Although the clinical impact of persistent lymphocytosis when ibrutinib is administered to CLL patients is not known, under the current International Workshop on CLL clinical criteria, treated patients have "delayed" responses. Combining ibrutinib with monoclonal antibodies in CLL has been hypothesized to accelerate and improve these responses.

Ibrutinib has been studied in combination with rituximab in one recently presented study. Rituximab is a chimeric/ human anti-CD20 monoclonal antibody that has been widely studied in the treatment of CLL as monotherapy and in combination with chemotherapy, with proven efficacy. ${ }^{47-50}$

Forty high-risk CLL patients were included in a singlecenter Phase II study of ibrutinib in combination with rituximab, in which high-risk was defined as treated or untreated CLL with 17p deletion/TP53 mutation, relapsed CLL with 11q deletion, and/or progression-free survival less than 36 months after chemoimmunotherapy. ${ }^{51}$ High-risk CLL features included $17 \mathrm{p}$ deletion or presence of TP53 mutation in $19 / 40$ patients, nonmutated IgVH in $31 / 40$ cases, and $11 \mathrm{q}$ deletion in 13/40 cases. The patients received ibrutinib $420 \mathrm{mg}$ per day orally in combination with rituximab $375 \mathrm{mg} / \mathrm{m}^{2}$ weekly during weeks $1-4$ (cycle 1 ), followed by monthly rituximab until cycle 6 , followed by continuation of ibrutinib until disease progression. At a median follow-up of four months, 20 patients were evaluable for response. The overall response rate was $85 \%$, with three cases of partial remission and persistent lymphocytosis. These findings suggest that the peak of lymphocytosis was earlier than when ibrutinib is used as a single agent. The treatment was well tolerated, with mostly grade 1 to 2 adverse events, and only 13 cases of grade 3 or 4 toxicity, including neutropenia, fatigue, pneumonia, and bone pain. ${ }^{51}$

Ibrutinib has also been studied in combination with ofatumumab, a fully humanized anti-CD20 monoclonal antibody. This antibody is approved for the treatment of fludarabine-refractory CLL based on an overall response rate of $45 \%$ as a single agent, and it has also been studied in combination with chemotherapy, with exciting results. ${ }^{50,52,53}$

The Phase Ib/II study of ibrutinib and ofatumumab included patients with relapsed/refractory CLL/small lymphocytic lymphoma who had received at least two prior therapies, including purine nucleoside analogs. Patients received ibrutinib $420 \mathrm{mg}$ daily on a 28-day cycle until disease progression. Ofatumumab was administered at a dose of $300 \mathrm{mg}$ on day 1 in cycle 2 and then as $2000 \mathrm{mg}$ on days 8,15 , and 22 of cycle 2 and on days 1,8 , and 15 of cycle 3 , and on day 1 in cycles $4-8$. Twenty-seven patients were enrolled, including three patients with Richter's transformation, and who had already received at least six cycles at the time of evaluation. Poor risk features included 17 p deletion (10/27), 11q deletion (9/20) purine nucleoside analog resistance (11/20), and bulky disease (15/27). After a median follow-up of 6.5 months, the overall response rate was $100 \%$ (one complete response) in the CLL/small lymphocytic lymphoma group, and two of three patients with Richter's transformation achieved partial remission. The most common side effects were grade 1 to 2 in severity. Grade 3 to 4 side effects included anemia (11\%), pneumonia (11\%), and urinary tract infection (7\%). ${ }^{54}$ The treatment was well tolerated and highly active in patients with refractory CLL/ small lymphocytic lymphoma.

\section{Ibrutinib and chemoimmunotherapy}

The current standard of care for fit and well patients with CLL is a combination of chemotherapy and anti-CD20 
monoclonal antibodies. Two regimens have become the mainstay of treatment for CLL, ie, FCR (fludarabine, cyclophosphamide, rituximab) and BR (bendamustine and rituximab). Both regimens have demonstrated significant activity and improved survival outcomes in the refractory as well as newly diagnosed CLL setting., ${ }^{2,55,56}$ Therefore, the possibility of using ibrutinib in combination with these regimens has been explored in a Phase Ib/II clinical trial in relapsed/refractory CLL patients. This study included 30 patients with relapsed/refractory CLL who had received 1-3 prior regimens. The FCR arm was closed to enrolment due to difficulty in accruing fludarabine-naïve CLL patients. The treatment regimen consisted of ibrutinib $420 \mathrm{mg}$ /day administered continuously in combination with BR (bendamustine $70 \mathrm{mg} / \mathrm{m}^{2}$ on days 1-2 with rituximab $375 \mathrm{mg} / \mathrm{m}^{2}$ in cycle 1 escalating to $500 \mathrm{mg} / \mathrm{m}^{2}$ in cycles $\left.2-6\right) .{ }^{57}$ Thirty-seven percent and $13 \%$ of patients were considered resistant to purine analogs and bendamustine, respectively (refractoriness was defined as a $<12$-month treatment-free interval). Patient characteristics and risk factors included bulky disease (53\%), Rai stage III/IV (47\%), presence of $17 \mathrm{p}$ deletion (23\%), and $11 \mathrm{q}$ deletion (43\%). After a median follow-up of 8.1 months, the overall response rate was $93 \%$, with $13 \%$ being complete responses, and estimated 11 -month progression-free survival was $90 \%$. Two patients developed progressive disease and discontinued treatment. These results appear to indicate a better clinical outcome than using BR alone, for which the overall response rate was $59 \%{ }^{56}$

Ibrutinib in combination with BR was well tolerated, with $23 \%$ of patients requiring reduction of their bendamustine dose. Toxicity was manageable, and consisted with diarrhea, nausea, fatigue, and skin rash. Grade 3 hematologic toxicity was present in $17 \%$ of patients (grade 4 in 10\%) and consisted mainly of neutropenia. Two patients developed tumor lysis syndrome. No discontinuations for adverse events were reported. Ninety percent of patients remained in the study.

There are Phase III clinical trials currently under way testing the efficacy of ibrutinib \pm bendamustine and rituximab (RESONATE), and ibrutinib versus ofatumumab (HELIOS) in relapsed/refractory CLL. RESONATE 2 will compare ibrutinib versus chlorambucil in the upfront setting in patients for whom chemotherapy is otherwise contraindicated. The outcomes of these Phase III studies will likely pave the way for US Food and Drug Administration approval of ibrutinib in the management of CLL. The feasibility of combination with immunomodulating agents, such as lenalidomide, may need to be explored further, especially in the setting of highrisk patients with $17 \mathrm{p}$ deletion.

\section{Future directions}

With the introduction of ibrutinib and other BCR-targeted therapies, and the exciting results in these studies mentioned here, we foresee that the therapeutic scenario for CLL will dramatically change in the years to come. Given its excellent toxicity profile, ibrutinib could be an appropriate choice for CLL patients in whom chemotherapy is otherwise contraindicated. This is generally due to their advanced age and/or concomitant comorbidities. Another group for which this therapy could be introduced includes patients with high-risk disease in whom chemoimmunotherapeutic approaches are ineffective. Further, ibrutinib can be envisaged as possible maintenance therapy for high-risk patients who have had a response to more conventional treatment. Finally, further investigations are needed to assess the place of ibrutinib in the setting of stem cell transplantation either as maintenance therapy or as a part of induction treatment prior to the procedure. We are witnessing a dynamic era in the therapeutic landscape for CLL, with active current studies in the pipeline and new trials in development. Their results are eagerly awaited.

\section{Disclosure}

The authors report no conflicts of interest in this work.

\section{References}

1. Ries L, Melbert D, Krapcho M, et al. SEER Cancer Statistics Review, 1975-2005. Bethesda, MD: National Cancer Institute; 2008.

2. Wierda $\mathrm{W}, \mathrm{O}$ 'Brien $\mathrm{S}$, Wen $\mathrm{S}$, et al. Chemoimmunotherapy with fludarabine, cyclophosphamide, and rituximab for relapsed and refractory chronic lymphocytic leukemia. J Clin Oncol. 2005; 23(18):4070-4078.

3. Keating MJ, O'Brien S, Albitar M, et al. Early results of a chemoimmunotherapy regimen of fludarabine, cyclophosphamide, and rituximab as initial therapy for chronic lymphocytic leukemia. J Clin Oncol. 2005;23(18):4079-4088.

4. Byrd JC, Rai K, Peterson BL, et al. Addition of rituximab to fludarabine may prolong progression-free survival and overall survival in patients with previously untreated chronic lymphocytic leukemia: an updated retrospective comparative analysis of CALGB 9712 and CALGB 9011. Blood. 2005;105(1):49-53.

5. Keating MJ, Cazin B, Coutre S, et al. Campath-1H treatment of T-cell prolymphocytic leukemia in patients for whom at least one prior chemotherapy regimen has failed. J Clin Oncol. 2002;20(1):205-213.

6. Chiorazzi N, Rai KR, Ferrarini M. Chronic lymphocytic leukemia. N Engl J Med. 2005;352(8):804-815.

7. Wiestner A. Emerging role of kinase-targeted strategies in chronic lymphocytic leukemia. Blood. 2012;120(24):4684-4691.

8. Klein U, Dalla-Favera R. New insights into the pathogenesis of chronic lymphocytic leukemia. Semin Cancer Biol. 2010;20(6):377-383.

9. Garcia-Munoz R, Galiacho VR, Llorente L. Immunological aspects in chronic lymphocytic leukemia (CLL) development. Ann Hematol. 2012;91(7):981-996.

10. Deaglio S, Malavasi F. Chronic lymphocytic leukemia microenvironment: shifting the balance from apoptosis to proliferation. Haematologica. 2009;94(6):752-756.

11. Stevenson FK, Caligaris-Cappio F. Chronic lymphocytic leukemia: revelations from the B-cell receptor. Blood. 2004;103(12):4389-4395. 
12. Fuentes-Panana EM, Bannish G, Monroe JG. Basal B-cell receptor signaling in B lymphocytes: mechanisms of regulation and role in positive selection, differentiation, and peripheral survival. Immunol Rev. 2004;197:26-40.

13. Woyach JA, Johnson AJ, Byrd JC. The B-cell receptor signaling pathway as a therapeutic target in CLL. Blood. 2012;120(6):1175-1184.

14. Davids MS, Brown JR. Targeting the B cell receptor pathway in chronic lymphocytic leukemia. Leuk Lymphoma. 2012;53(12):2362-2370.

15. Packham G, Stevenson F. The role of the B-cell receptor in the pathogenesis of chronic lymphocytic leukaemia. Semin Cancer Biol. 2010;20(6):391-399.

16. Dal Porto JM, Gauld SB, Merrell KT, Mills D, Pugh-Bernard AE, Cambier J. B cell antigen receptor signaling 101. Mol Immunol. 2004; 41(6-7):599-613.

17. Burger JA, Ghia P, Rosenwald A, Caligaris-Cappio F. The microenvironment in mature B-cell malignancies: a target for new treatment strategies. Blood. 2009;114(16):3367-3375.

18. Burger JA. Nurture versus nature: the microenvironment in chronic lymphocytic leukemia. Hematology Am Soc Hematol Educ Program. 2011;2011:96-103.

19. Monroe JG. ITAM-mediated tonic signalling through pre-BCR and BCR complexes. Nat Rev Immunol. 2006;6(4):283-294.

20. Rui L, Schmitz R, Ceribelli M, Staudt LM. Malignant pirates of the immune system. Nat Immunol. 2011;12(10):933-940.

21. Quiroga MP, Balakrishnan K, Kurtova AV, et al. B-cell antigen receptor signaling enhances chronic lymphocytic leukemia cell migration and survival: specific targeting with a novel spleen tyrosine kinase inhibitor, R406. Blood. 2009;114(5):1029-1037.

22. Hoellenriegel J, Coffey GP, Sinha U, et al. Selective, novel spleen tyrosine kinase (Syk) inhibitors suppress chronic lymphocytic leukemia B-cell activation and migration. Leukemia. 2012;26(7): $1576-1583$.

23. Lannutti BJ, Meadows SA, Herman SE, et al. CAL-101, a p110delta selective phosphatidylinositol-3-kinase inhibitor for the treatment of B-cell malignancies, inhibits PI3K signaling and cellular viability. Blood. 2011;117(2):591-594.

24. Kahl B, Byrd J, Flinn I, et al. Clinical safety and activity in a phase 1 study of CAL-101, an isoform-selective inhibitor of phosphatidylinositol 3-kinase P110 \{delta\}, in patients with relapsed or refractory non-Hodgkin lymphoma. Blood. 2010;116:741.

25. Coutre S, Byrd J, Furman R, Brown J, Benson D, Wagner-Johnston N. Phase I study of CAL-101, an isoform-selective inhibitor of phosphatidylinositol 3-kinase P110d, in patients with previously treated chronic lymphocytic leukemia. J Clin Oncol. 2011;29:451s.

26. Smith CI, Baskin B, Humire-Greiff P, et al. Expression of Bruton's agammaglobulinemia tyrosine kinase gene, BTK, is selectively down-regulated in T lymphocytes and plasma cells. J Immunol. 1994; 152(2):557-565.

27. de Weers M, Mensink RG, Kraakman ME, Schuurman RK, Hendriks RW. Mutation analysis of the Bruton's tyrosine kinase gene in X-linked agammaglobulinemia: identification of a mutation which affects the same codon as is altered in immunodeficient xid mice. Hum Mol Genet. 1994;3(1):161-166.

28. Conley ME, Dobbs AK, Farmer DM, et al. Primary B cell immunodeficiencies: comparisons and contrasts. Annu Rev Immunol. 2009;27:199-227.

29. Afar DE, Park H, Howell BW, Rawlings DJ, Cooper J, Witte ON. Regulation of Btk by Src family tyrosine kinases. Mol Cell Biol. 1996; 16(7):3465-3471.

30. Winer ES, Ingham RR, Castillo JJ. PCI-32765: a novel Bruton's tyrosine kinase inhibitor for the treatment of lymphoid malignancies. Expert Opin Investig Drugs. 2012;21(3):355-361.

31. Buggy JJ, Elias L. Bruton tyrosine kinase (BTK) and its role in B-cell malignancy. Int Rev Immunol. 2012;31(2):119-132.

32. Islam TC, Smith CI. The cellular phenotype conditions Btk for cell survival or apoptosis signaling. Immunol Rev. 2000;178:49-63.
33. Honigberg LA, Smith AM, Sirisawad M, et al. The Bruton tyrosine kinase inhibitor PCI-32765 blocks B-cell activation and is efficacious in models of autoimmune disease and B-cell malignancy. Proc Natl Acad Sci U S A. 2010;107(29):13075-13080.

34. Davis RE, Ngo VN, Lenz G, et al. Chronic active B-cellreceptor signalling in diffuse large B-cell lymphoma. Nature. 2010;463(7277):88-92.

35. Herman SE, Gordon AL, Hertlein E, et al. Bruton tyrosine kinase represents a promising therapeutic target for treatment of chronic lymphocytic leukemia and is effectively targeted by PCI-32765. Blood. 2011;117(23):6287-6296.

36. Ponader S, Chen SS, Buggy JJ, et al. The Bruton tyrosine kinase inhibitor PCI-32765 thwarts chronic lymphocytic leukemia cell survival and tissue homing in vitro and in vivo. Blood. 2012;119(5):1182-1189.

37. de Rooij MF, Kuil A, Geest CR, et al. The clinically active BTK inhibitor PCI-32765 targets B-cell receptor- and chemokine-controlled adhesion and migration in chronic lymphocytic leukemia. Blood. 2012; 119(11):2590-2594.

38. Advani R, Sharman J, Smith S, et al. The Btk inhibitor PCI-32765 is highly active and well tolerated in patients with relapsed/refractory B cell malignancies: final results from a phase I study. Ann Oncol. 2011;22 Suppl 4:135.

39. Byrd J, Blum K, Burger J, et al. Activity and tolerability of the Bruton's tyrosine kinase (Btk) inhibitor PCI-32765 in patients with chronic lymphocytic leukemia/small lymphocytic lymphoma (CLL/SLL): Interim results of a phase Ib/II study. J Clin Oncol. 2011;29(15):6508.

40. Byrd JC, Furman RR, Coutre SE, et al. The Bruton's tyrosine kinase (BTK) inhibitor PCI-32765 (P) in treatment-naive (TN) chronic lymphocytic leukemia (CLL) patients (pts): interim results of a phase Ib/II study. ASCO Meeting Abstracts. 2012;30(Suppl 15):6507.

41. Byrd JC, Furman RR, Coutre S, et al. The Bruton's tyrosine kinase (BTK) inhibitor ibrutinib (PCI-32765) promotes high response rate, durable remissions, and is tolerable in treatment naive (TN) and relapsed or refractory (RR) chronic lymphocytic leukemia (CLL) or small lymphocytic lymphoma (SLL) patients including patients with high-risk (HR) disease: new and updated results of 116 patients in a Phase Ib/II study. ASH Annual Meeting Abstracts. 2012;120(21):189.

42. O'Brien S, Burger JA, Blum KA, et al. The Bruton's tyrosine kinase (BTK) inhibitor PCI-32765 induces durable responses in relapsed or refractory $(\mathrm{R} / \mathrm{R})$ chronic lymphocytic leukemia/small lymphocytic lymphoma (CLL/SLL): follow-up of a Phase Ib/II study. ASH Annual Meeting Abstracts. 2011;118(21):983.

43. Hallek M, Cheson BD, Catovsky D, et al. Guidelines for the diagnosis and treatment of chronic lymphocytic leukemia: a report from the International Workshop on Chronic Lymphocytic Leukemia updating the National Cancer Institute Working Group 1996 guidelines. Blood. 2008; 111(12):5446-5456

44. Cheson BD, Byrd JC, Rai KR, et al. Novel targeted agents and the need to refine clinical end points in chronic lymphocytic leukemia. $J$ Clin Oncol. 2012;30(23):2820-2822.

45. Friedberg JW, Sharman J, Sweetenham J, et al. Inhibition of Syk with fostamatinib disodium has significant clinical activity in nonHodgkin lymphoma and chronic lymphocytic leukemia. Blood. 2010; 115(13):2578-2585.

46. Hoellenriegel J, Meadows SA, Sivina M, et al. The phosphoinositide 3'-kinase delta inhibitor, CAL-101, inhibits B-cell receptor signaling and chemokine networks in chronic lymphocytic leukemia. Blood. 2011;118(13):3603-3612.

47. O'Brien SM, Kantarjian H, Thomas DA, et al. Rituximab doseescalation trial in chronic lymphocytic leukemia. J Clin Oncol. 2001; 19(8):2165-2170.

48. Byrd JC, Murphy T, Howard RS, et al. Rituximab using a thrice weekly dosing schedule in B-cell chronic lymphocytic leukemia and small lymphocytic lymphoma demonstrates clinical activity and acceptable toxicity. J Clin Oncol. 2001;19(8):2153-2164. 
49. Byrd JC, Peterson BL, Morrison VA, et al. Randomized phase 2 study of fludarabine with concurrent versus sequential treatment with rituximab in symptomatic, untreated patients with B-cell chronic lymphocytic leukemia: results from Cancer and Leukemia Group B 9712 (CALGB 9712). Blood. 2003;101(1):6-14.

50. Jaglowski SM, Alinari L, Lapalombella R, Muthusamy N, Byrd JC. The clinical application of monoclonal antibodies in chronic lymphocytic leukemia. Blood. 2010;116(19):3705-3714.

51. Burger JA, Keating MJ, Wierda WG, et al. The BTK inhibitor ibrutinib (PCI-32765) in combination with rituximab is well tolerated and displays profound activity in high-risk chronic lymphocytic leukemia (CLL) patients. ASH Annual Meeting Abstracts. 2012;120(21):187.

52. Coiffier B, Lepretre S, Pedersen LM, et al. Safety and efficacy of ofatumumab, a fully human monoclonal anti-CD20 antibody, in patients with relapsed or refractory B-cell chronic lymphocytic leukemia: a phase 1-2 study. Blood. 2008;111(3):1094-1100.

53. Wierda WG, Padmanabhan S, Chan GW, Gupta IV, Lisby S, Osterborg A. Ofatumumab is active in patients with fludarabinerefractory CLL irrespective of prior rituximab: results from the phase 2 international study. Blood. 2011;118(19):5126-5129.

54. Jaglowski SM, Jones JA, Flynn JM, et al. A phase Ib/II study evaluating activity and tolerability of BTK inhibitor PCI-32765 and ofatumumab in patients with chronic lymphocytic leukemia/small lymphocytic lymphoma (CLL/SLL) and related diseases. ASCO Meeting Abstracts. 2012;30(Suppl 15):6508.
55. Hallek M, Fischer K, Fingerle-Rowson G, et al. Addition of rituximab to fludarabine and cyclophosphamide in patients with chronic lymphocytic leukaemia: a randomised, open-label, phase 3 trial. Lancet. 2010; 376(9747):1164-1174.

56. Fischer K, Cramer P, Busch R, et al. Bendamustine combined with rituximab in patients with relapsed and/or refractory chronic lymphocytic leukemia: a multicenter phase II trial of the German Chronic Lymphocytic Leukemia Study Group. J Clin Oncol. 2011; 29(26):3559-3566.

57. Brown J, Barrientos J, Flinn I, Barr P, Burger J, Navarro T. The Bruton's tyrosine kinase (BTK) inhibitor ibrutinib combined with bendamustine and rituximab is active and tolerable in patients with relapsed/refractory CLL, interim results of a phase IB/II study. Abstract presented at the European Hematology Association meeting, Amsterdam, The Netherlands, June 14-17, 2012.

\section{Core Evidence}

\section{Publish your work in this journal}

Core Evidence is an international, peer-reviewed open-access journal evaluating the evidence underlying the potential place in therapy of drugs throughout their development lifecycle from preclinical to postlaunch. The focus of each review is to evaluate the case for a new drug or class in outcome terms in specific indications and patient groups.

\section{Dovepress}

The manuscript management system is completely online and includes a very quick and fair peer-review system, which is all easy to use. Visit http://www.dovepress.com/testimonials.php to read real quotes from published authors. 